\title{
The Construction by Computer of a Diagnostic Key to the Genera of Yeasts and Other Such Groups of Taxa
}

\author{
By R. W. PAYNE, ${ }^{*}$ D. YARROW ${ }^{2}$ AND J. A. BARNETT ${ }^{3}$ \\ ${ }^{1}$ Rothamsted Experimental Station, Harpenden, Hertfordshire AL5 2JQ, U.K. \\ ${ }^{2}$ Centraalbureau voor Schimmelcultures, Yeast Division, Laboratory for Microbiology, \\ Julianalaan 67A, $2628 \mathrm{BC}$ Delft, The Netherlands \\ ${ }^{3}$ School of Biological Sciences, University of East Anglia, Norwich NR4 7TJ, U.K.
}

(Received 3 February 1982)

Groups of taxa such as genera, or groups derived from some forms of cluster analysis, may have insufficient test results that are constant within the groups to allow diagnostic keys and tables to be constructed in the usual way. This paper describes how the usual methods can be adapted to allow construction based on information about the individual group members, instead of on the overall group information. A new key to the genera of yeasts is constructed by these modified methods.

\section{INTRODUCTION}

This paper reports new methods for computing diagnostic keys and tables to identify the group of taxa to which a specimen belongs, rather than the taxon itself. These methods are of general interest for microbial taxonomy and are illustrated below by the construction of a key to the genera of yeasts.

Barnett \& Pankhurst (1974) and Barnett et al. (1979) devised new keys to yeast species. These keys were constructed by computer programs which required, for data, a species $\times$ test table, each entry indicating the results that can occur when a test is used with a particular species. An entry was coded (i) as positive, if all yeasts of that species always give a positive response to the test, (ii) as negative, if all yeasts of the species always give a negative response, or (iii) as variable (or equivocal), if the response might differ between strains of a species, or be particularly dependent on the precise method of testing, so that sometimes the result might appear positive and sometimes negative.

It might seem that a key to genera could be constructed similarly, by forming a genus $x$ test table of results, and then using the same methods as those for the keys to the species. However, in such a table of results for the genera of yeasts, $45 \%$ of the entries were variable, and 133 pairs of genera could not be distinguished by this overall summary. The results are unsuitable for distinguishing between the genera, although suitable for the species, for two main reasons. First, most of these results are from nutritional tests, whilst the genera are mostly not classified with respect to their nutritional characteristics. Secondly, many characteristics important for classifying species into genera are inconvenient to use for routine identification and, hence, were not used in the battery of features on which the key to the species was based (Barnett et al., 1979). For example, Debaryomyces is distinguished from Pichia by the former producing ascospores with warty walls and the latter producing ascospores with smooth walls. However, some species of Pichia have ascospores with warty walls; but, unlike those of Debaryomyces, electron microscopy has revealed that these warts are formed exclusively by the outer layer of the ascospore wall (Kreger-van Rij, 1970a,b).

Thus, the difficulties in constructing keys to yeast genera arise because of the need to use different characteristics for identifying from those used for classifying the genera. However, 
Table 1. Results for taxa A to I with tests 1 to 6

\begin{tabular}{|c|c|c|c|c|c|c|c|c|}
\hline \multirow[b]{2}{*}{ Taxon } & & \multicolumn{6}{|c|}{ Tests } & \\
\hline & Group & 1 & 2 & 3 & 4 & 5 & 6 & \\
\hline A & I & - & + & - & - & - & + & \multirow{9}{*}{$\begin{array}{l}\text { Results for } \\
\text { each taxon }\end{array}$} \\
\hline B & I & + & - & - & - & $\mathrm{v}$ & + & \\
\hline C & I & + & + & - & + & - & + & \\
\hline D & II & - & + & - & + & + & + & \\
\hline E & II & + & + & + & + & + & $\mathrm{v}$ & \\
\hline$F$ & II & - & $\mathrm{v}$ & + & + & + & + & \\
\hline $\mathrm{G}$ & III & - & - & - & + & - & + & \\
\hline $\mathrm{H}$ & III & - & - & - & $\mathrm{v}$ & + & - & \\
\hline I & III & - & - & + & + & - & - & \\
\hline & I & $\mathrm{v}$ & $\mathrm{v}$ & - & $\mathrm{v}$ & $v$ & + & \multirow{3}{*}{$\begin{array}{l}\text { Summarized } \\
\text { results for each } \\
\text { group of taxa }\end{array}$} \\
\hline & II & $\mathrm{v}$ & $\mathrm{v}$ & $\mathrm{v}$ & + & + & $\mathrm{v}$ & \\
\hline & III & - & - & v & $\mathrm{v}$ & 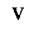 & $\mathrm{v}$ & \\
\hline
\end{tabular}

problems can occur even when the same characteristics are used for both. For example, many numerical methods of classification obtain a measure of the similarity of each pair of taxa and then form groups, or clusters, by merging similar taxa. Because the similarity measure is a single figure, based on all the characteristics of the pair of taxa concerned, there is no guarantee that any of the characteristics will be other than variable for the groups formed.

Maximal predictive classification (Gower, 1973, 1974; Barnett et al., 1975) is not based on pairwise similarity, but aims to construct groups such that knowing the group to which a taxon belongs enables the maximum number of correct predictions to be made about that taxon. However, even maximal predictive groups may sometimes have few non-variable characteristics. As an example, Table 1 shows a set of hypothetical characteristics for taxa A to I and binary tests 1 to 6 ; the second column of the table contains the group number of each taxon for a maximal predictive classification into three groups; the group characteristics are summarized in the last three lines of the table.

This paper shows how these two difficulties, namely, (i) the disassociation of identifying and classifying and (ii) the presence of many variable results, may be overcome.

\section{METHODS}

Irredundant test sets. To identify yeasts it is usually impracticable to use tests sequentially, that is, to do each test only after interpreting the results of previous tests. Accordingly, Barnett et al. (1979) assumed that all the tests required for a given key would be done simultaneously. As the full set of tests in the key will be done for any identification, that set should be minimal. Hence it should contain no redundant tests: for example, test 5 in Table 1 is redundant, as it can be omitted without making any taxon unidentifiable. However, the set of tests 12346 is termed irredundant (with regard to the identification of individual taxa), since if any further tests are omitted there will be at least one pair of taxa that can no longer be distinguished. For example, if test 1 is also omitted, taxa $C$ and $\mathrm{D}$ cannot be distinguished.

Barnett et al. (1979) used a method (reviewed by Payne \& Preece, 1980) that determined all irredundant test sets available to identify the yeast species in a particular key. Appendix 1 describes how to adapt this method to form sets to identify groups instead of individual taxa, and shows that there are five irredundant sets of tests to identify the groups in Table 1, namely, 12346, 1456, 2456, 1235 and 1256. Each set can distinguish between all pairs of taxa belonging to different groups, but not necessarily between pairs of taxa in the same group. For example, there is no test in set 1456 to distinguish taxon D from taxon F, both of which are in group II.

Diagnostic keys and tables. To enable the specimens to be identified, given their results for the tests in the chosen irredundant set, a diagnostic table may be printed. Table 2 shows a diagnostic table for identifying the groups of taxa in Table 1, based on the second irredundant set, tests 1456.

An alternative means of identification is the diagnostic (or identification) key. This is most commonly used in situations when tests are done sequentially. However, as described above, it is equally possible to use a key with 
Table 2. Diagnostic table for the groups of taxa in Table 1, based on tests 1, 4, 5 and 6

$\begin{array}{ccccc}\overbrace{1} & 4 & 5 & 6 & \text { Group (Taxon) } \\ + & + & + & +/- & \text { II (E) } \\ + & + & - & + & \text { I (C) } \\ + & - & +/- & + & \text { I (B) } \\ - & + & + & + & \text { II (D or F) } \\ - & + & + & - & \text { III (H) } \\ - & + & - & +/- & \text { III (G or I) } \\ - & - & + & - & \text { III (H) } \\ - & - & - & + & \text { I (A) }\end{array}$

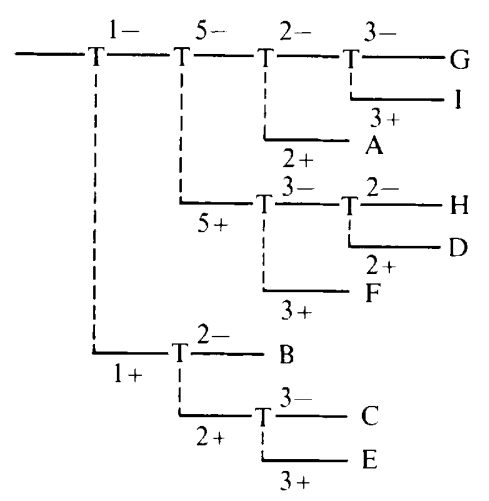

Fig. 1. Key to the individual taxa A to I in Table 1, based on tests (T) 1, 2, 3 and 5 .

non-sequential testing and, if many test results are variable, such a key will be more compact than the corresponding diagnostic table (Payne \& Preece, 1980).

An example, for identifying the individual taxa in Table 1, is shown diagrammaticaly in Fig. 1. In this key, test 1 is first, followed by test 5 for a negative result, test 2 for a positive result and so on. Thus, specimens of taxon $B$ would be identified by test $1+$, test $2-$. Such a sequence of test results is termed a branch of the key.

Several computer programs have been devised for constructing keys to taxa (e.g. Pankhurst, 1970; Morse, 1971; Dallwitz, 1974; Payne, 1975). These all operate similarly, selecting first the test that best divides the taxa into subsets. For a binary test, there are two subsets: the first containing the taxa not eliminated if a negative result is observed with the test (i.e. the taxa with either negative or variable results), and the second subset containing the taxa not eliminated by a positive result. The programs then select the best test to use with each subset, continuing until the subsets each contain only one taxon. The best test is usually taken as that with minimum value of some selection criterion function, a function involving the following: $m_{i}$, the number of possible results to test $i ; p_{i k}$, the proportion of taxa in the current subset that always give result $k$ to test $i$; and $r_{i}$, the proportion of taxa in the current subset with variable results to test $i$. The proportions are often weighted by the frequencies with which the taxa are expected to occur.

Keys to identify groups of taxa. It is usually wasteful to use a key like that in Fig. 1 and identify the group by first identifying the individual taxon. For example, after test $1-$, test $5-$ and test $2-$ in Fig. 1 , the taxa not eliminated, G and I, both belong to group III; so test 3 is unnecessary. This suggests one modification to the usual method of key construction, namely, that each branch should now terminate when the taxa are all from the same group.

Selection criterion functions, designed to select tests to identify individual taxa, are usually unsuitable for selecting tests to identify groups of taxa. They require modification to take account of the fact that there is no need to separate taxa belonging to the same group. Appendix 2 explains how this can be done and derives two new functions, $G_{\mathrm{v}}$ and $G_{\mathrm{e}}$. 


\section{Table 3. Irredundant test sets to identify yeast genera}

These are sets of tests that separate the genera as far as possible, and are minimal in the sense that, if any test is deleted from the set, there will be some pair of species in different genera that can no longer be distinguished. The tests are taken from those listed by Barnett et al. (1979).

Six sets are presented below, formed by taking all 43 tests in group A and one test from each of groups $\mathrm{B}$ and $\mathrm{C}$.

Group $A$

1 D-Glucose fermentation

3 Maltose fermentation

6 Trehalose fermentation

12 D-Galactose growth

13 L-Sorbose growth

14 D-Ribose growth

18 L-Rhamnose growth

19 Sucrose growth

20 Maltose growth

21 Trehalose growth

26 Melibiose growth

27 Lactose growth

28 Raffinose growth

29 Melezitose growth

30 Inulin growth

31 Starch growth

32 Glycerol growth

33 Erythritol growth

35 Galactitol growth

36 D-Mannitol growth

37 D-Glucitol growth

38 myo-Inositol growth
$39 \mathrm{D}-\delta$-Gluconolactone growth

40 2-Ketogluconate growth

44 Citrate growth

47 Ethylamine growth

49 Nitrate growth

54 Growth without biotin

55 Growth without thiamin

56 Growth without pyridoxine

57 Growth without niacin

60 Growth in 50\% D-glucose

61 Growth in $60 \% \mathrm{D}$-glucose

63 Growth with $0.01 \%$ cycloheximide

65 Starch formation

66 Pink colonies

67 Budding cells

68 Splitting cells

69 Apical budding

72 Filamentous

74 Septate hyphae

75 Ascosporogenous

76 Ascospores spherical, oval or reniform
Group $B$

23 Cellobiose growth

24 Salicin growth

25 Arbutin growth

Group C

42 DL-Lactate growth

43 Succinate growth

\section{RESULTS AND DISCUSSION}

The methods described above have been incorporated into the computer program Genkey (Payne, 1975, 1978) and applied to the data of Barnett et al. (1979). Table 3 lists the six smallest irredundant test sets for identifying the genera of yeasts, each set containing 45 tests. There were 716 sets in all to choose from, containing up to 52 tests.

A key to the genera based on the irredundant set containing all 43 tests in group A of Table 3 , together with tests 23 and 42, was constructed using selection criterion $G_{\mathrm{e}}$ and is printed in Table 4 is one of the compact forms of Payne et al. (1974). This key includes the results of physiological tests, some morphological tests and tests for ascospores. If the results of testing for ascospores are omitted there are six irredundant sets, each containing 54 tests, and a key based on one of these sets, constructed similarly to that in Table 4, took 581 lines (compared to the 307 lines in Table 4). Also, the number of pairs of species in different genera that cannot be distinguished rises from 32 to 140 .

The key in Table 4 thus provides a compromise between including (i) solely physiological characteristics, as in the key of Barnett \& Pankhurst (1974) and some of those of Barnett et al. (1979), and (ii) the varied features used by Lodder (1970) in her key to the genera. Lodder (1970) included, amongst others, the formation of ballistospores and their shape, and the presence of clamp connections, as well as some more complex features that are difficult to observe, excessively subjective or awkward to interpret. These are exemplified by the following: (i) "cells often "ogival"; strong acetic acid production from glucose; characteristic aroma; cells on malt agar short-lived'; (ii) 'ascospores hat- or helmet-shaped, or apparently globose with an indistinct ledge, not conjugating in pairs'.

In order to diagnose a yeast's genus, Lodder's key to the genera uses about the same number of steps as that in Table 4. Although her key involves only about 12 kinds of examination in the laboratory, the scrutiny of asci, ascospores and filaments is in much 
Table 4. Key to the genera of yeasts

The genera are those accepted by Barnett et al. (1979).

Negative

Positive

1 Ascosporogenous

Pink colonies

3 Filamentous

4 myo-Inositol growth - -

5 Budding cells

6 Maltose growth

Lactose growth

8 Apical budding - - -

L-Sorbose growth

D-Glucose fermentation-

\section{Nitrate growth}

Cellobiose growth

Growth without biotin -

Sucrose growth
D-Mannitol growth - -

D-Mannitol growth

D, L-Lactate growth

D-Glucose fermentation_

Cellobiose growth

Starch formation - -

Erythritol growth

Lactose growth

Cellobiose growth

Melezitose growth - -

Melibiose growth__ -

Starch growth

Raffinose growth

D-Glucose fermentation -

Nitrate growth

D, L-Lactate growth -

D-Galactose growth - -

Raffinose growth

Budding cells

Maltose growth ${ }^{-}--$

Melezitose growt $\bar{h}$

Glycerol growth _...

Nitrate growth $\ldots$

Lactose growth

Raffinose growth

Starch formation _ -

Sucrose growth

Melibiose growth_.

Splitting cells

Apical budding

myo-Inositol growth - -

Budding cells _

Erythritol growth $\overline{\text { L }}$

D-Mannitol growth - -

Maltose fermentation - -

Nitrate growth

Trehalose growth
D-Mannitol growth

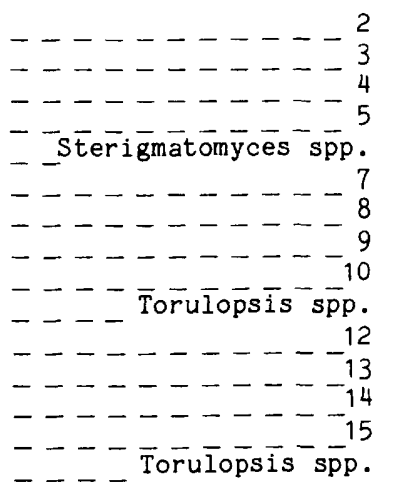

Candida sp. Törulopsis sp. Torulopsis spp. Trigonopsis sp. $\bar{S} c \bar{h} i \overline{z o b} l$ astosporion sp.

$$
\text { - }-\ldots \ldots-\ldots 21
$$$$
---\cdots----22
$$

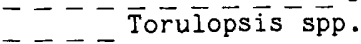$$
\text { - Torulopsis spp. }
$$$$
\text { _ - _ - _ _ _ _ } 26
$$$$
\text { - - - - - - - - - } 27
$$$$
\text { Torūopsis sp. }
$$$$
\text { - - - - - Bu] } 29
$$$$
\text { - }-\ldots \text { Bullera sp. }
$$$$
\text { - - - - Cañida } \overline{s p} \text {. }
$$$$
\text { - - - Torulopsis spp. }
$$$$
\text { - - Aessosporon sp. }
$$$$
\text { - Sterigmatomyces spp. }
$$$$
\text { _ _ Cryptococcus spp. }
$$$$
\text { - - - cryptococcus }-37
$$$$
\text { - - Cryptococcus spp. }
$$

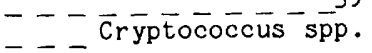$$
\text { - Cryptococcus spp. }
$$$$
\text { _ - Bullera sp. }
$$$$
\text { - Cryptococcus sp. }
$$$$
\text { _ - - Cryptococcus sp. }
$$$$
\text { - - Filobasidium sp. }
$$

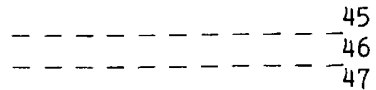

- Sterigmatomyces sp̄p.

- - - . - . - - $-\frac{49}{50}$

- - - - - - - 51

- - - - - - - - 52

- - - - - - - - - - -53

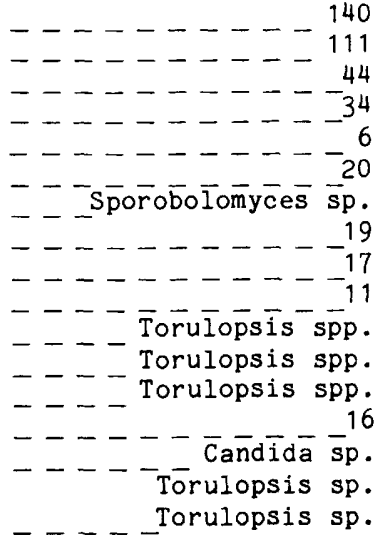

18

Toruīopsis $\overline{\text { spp. }}$. Torulopsis sp.

- - - - - $\ldots$ - 33

- - - - - - - 30

- - - . - . - 28

$---\ldots-\ldots--24$

- Torulopsis $\overline{\text { spp. }}$.

- - Torulopsis spp.

- - - Candida spp.

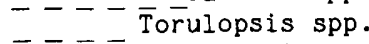

-

- _ - - _

- - - sēlēnozyma sp.

- - - $\bar{R}$ hodotorula sp.

- - - Cryptococcus sp.

-

- - - - - - - 36

- Fìlobasididum sp.

- - - . . . - -41

- - - B Būilera $\overline{\mathrm{sp}}$.

- - - - Bullera sp.

- - - - - - - - 43

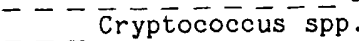

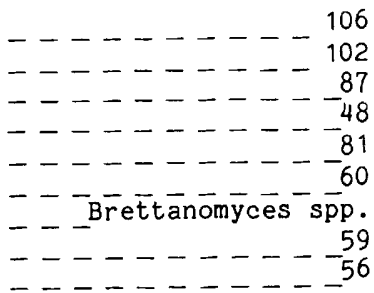


Table 4-cont.

Negative

Positive

53 Growth without_ _ _ pyridoxine

54 D-Glucose fermentation.

55 Cellobiose growth _ - -

56 L-Sorbose growth

57 Cellobiose growth

58 D-Galactose growth - -

59 D-Galactose growth

60 Lactose growth_ $\ldots \ldots$

61 Nitrate growth

62 Cellobiose growth

63 Sucrose growth

64 Galactitol growth - -

65 Citrate growth

66 Maltose fermentation -

67 Septate hyphae

68 D, L-Lactate growth - -

69 Melibiose growth - -

70 Starch growth

71 D-Galactose growth - -

72 Maltose fermentation - -

73 Raffinose growth _ _ _
L-Sorbose growth

Maltose fermentation- -

Trehalose growth

Raffinose growth

Cellobiose growth

L-Rhamnose growth

Sucrose growth

Septate hyphae - - -

Sucrose growth

Citrate growth

Growth without biotin -

Maltose fermentation

L-Rhamnose growth

Budding cells - -

Nitrate growth -

Melezitose growth -

Erythritol growth - -

Growth without biotin

D-Glucose fermentation-

Melibiose growth

Lactose growth - - -

Lactose growth

Erythritol growth

Starch formation

Erythritol growth

Starch growth

L-Rhamnose growt $\bar{h}-$

Septate hyphae

Nitrate growth - - -

Cellobiose growt $\bar{h}-\ldots$

Erythritol growth - -

Septate hyphae

Budding cells

Nitrate growth

Lactose growth
_. - . . . . . 54

- - - Torüropsis $\frac{55}{\mathrm{sp}}$.

- $\ldots$ - Torulopsis sp.

- - Bretettanomyces $\overline{s p}$.

Candida spp.

- - - - Candida spp.

- - - - - - -61

Candida sp̄p.

- ------64

$-----\cdots--66$

- - Bretténomyces $\overline{s p}$.

- - B

-

- - - -

- Candida $\overline{s p p}$.

. Candida sp.

_. . _ _ _ _ _ 73

_ _ _ Torulopsis sp.

Torulopsis sp.

- Ácículoconidium sp.

- - - Torulopsis $\overline{\text { spp }}$.

- - - - - Candida 79

_

Spōrob̄olomyces sp.

_ _ _ . _ _ _ 82

- - - - Candida spp.

- - - Candida sp.

- Candida spp.

- Tr i chosporon spp.

- $-\overline{\text { Stterigmatomyces sp. }}$

_ _ _ _ _ _ . _ 89

- - - - - - -99

-

- Candid̄a spp.

- - - - . . 93

- - - - Candida $\overline{\text { sp. }}$. cryptococcus sp.

_.

_ _ . _ _ _ _ _ _ -98

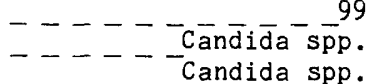

- Sterigmatomyces sp.

103

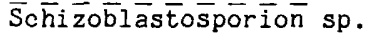
Torulopsis sp.

_. . Torulopsis sp.

- - Geotrichum spp.

Trichosporōon 108

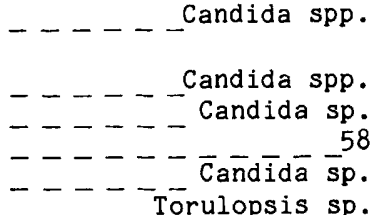

_Brettanomyces spp.

- - - - - - - 80

- - - - - - - - 76

- - - - - - - - -63

- $-1--67$

- -

Candida spp.

- Candida sp.

- - - 75

- - - C

- - - - - -74

--------74
--------71

_ $\ldots$ - Candida spp.

Candida sp.

- --- Candida sp.

- - - - Candida spp.

- - - - - 78

_ Candida spp.

- - - Torulopsis sp.

- $-{ }_{-}$Candida spp.

- - - - Candida $\overline{\text { spp }}$.

_. - _ - _ _ _ 84

Torūiopsis sp.

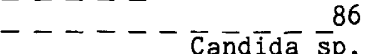

- - - - - Candida sp.

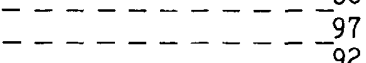

-

Filobasidium sp.

_ $-\ldots$ - Candida spp.

- - - - - $\overline{\text { and }}$ - -95

_ $\ldots$ - Candida spp.

$----\overline{\text { Cañdida }} \frac{96}{\mathrm{sp} .}$ C $\bar{r}$ yptococcus sp. Filobasidium sp.

100

-

- _ - _

- $-\ldots$ - Cañdida $\mathrm{sp.}$

- - - - - -105

- - Sympódiomyces sp.

- Leucosporidium spp.

- - - - _ - -107

- - - - - - -110

- - - - - - - - 109 
Table 4-cont.

Negative

110 D-Galactose growth

111 myo-Inositol growt $\bar{h}-$

112 Starch formation _ _ -

113 Nitrate growth _

114 Trehalose growth

115 L-Rhamnose growth

116 Galactitol growth - -

117 Starch growth

118 Filamentous

119 2-Ketogluconāte $\overline{\text { growth }} \overline{-}^{-}$
Maltose growth

D-Galactose growth

Raffinose growth

Erythritol growth Sucrose growth

Filamentous

Melezitose growth

Growth without thi amin-

Galactitol growth

Maltose growth

Septate hyphae_

Septate hyphae

Starch growth

Melezitose growt $\bar{h}$ Sucrose growth

L-Sorbose growth

Erythritol growth - -

Nitrate growth

Ascospores spherical, oval or reniform

Nitrate growth

Apical budding - - Apical budding $\overline{-}-$ L-Rhamnose growth _ _ Sucrose growth $\ldots$ Filamentous

Septate hyphāe_D-Glucitol growth - Trehalose growth - Erythritol growth - D-Mannitol growth - Filamentous Septate hyphāe Cellobiose growth - Starch growth _ _ _ Citrate growth D-Galactose growth Melezitose growth Growth without _ _ . pyridoxine

D-Galactose growth Raffinose growth 2-Ketogluconate growth

Growth without thiaminD-Glucitol growth - -

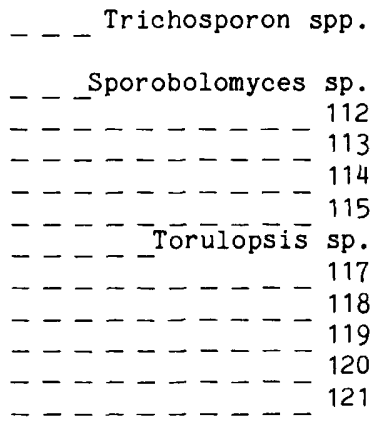

Rhodotorula sp. sporobolomyces sp. Rhodosporidium $\mathrm{sp}$.

_ - _ _ _ _ _ 124

- - - - - - 125

- - - - - - 126

_-

-

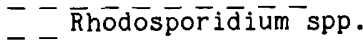

- $-\ldots \ldots+\ldots$

- - sp̄oñobōō̄yces 131

_ Spórob̄olomyces sp.

_- ${ }_{\text {- }}$ Rhodotorula $\mathrm{sp.}$.

_. Rh̆odosporidium sp.

Phaffia sp.

- - - . Phaffia sp. 139

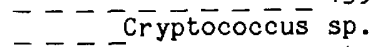

_ $\ldots \ldots \ldots 141$

$-\ldots-142$

- - - - - - -143

- - - - - - - - 144

- - - - - - 145

- - - - $-\overline{\text { Pichia }} \overline{\text { spp. }}$

- - Pichia spp.

_ $\ldots \ldots$ - Pichia spp.

- Säç̄ar omycōpsis sp.

_ $-\ldots \ldots$. $\ldots . .150$

- $\ldots$ - $-\ldots-151$

- - - Píchia sp.

_ $-\ldots$ - Dekkera spp.

154

- - Ne- -

_

- - - - - - - - - 157

- - - - - 158

- Sacchar omycopsis sp.

_ $\ldots \ldots$ _ $\ldots i c h i a$ sp.

Pichia spp.

_- Mets̄innikowia spp.
Positive

_ _ Sarcinosporon sp. Trichosporon sp. Trichosporon spp.

- - -

- - - - - - - - 137

- - - - - - -123

116

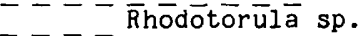

_ _ Rhodotorula spp.

- _ Sporobolomyces sp.

122

- - _ Rh̆odotō̄ūa spp. Rhodotorula sp.

sporobolomyces sp. Rhodotorula spp.

Rhodotorula sp. - - Rhodotorula sp.

-

- - - - - - 127

- Sporōobolomyces spp.

- - - - $-\ldots$ - -132

- - - sp̄oridibolus sp.

_

Aessosporon sp.

sporobolomyces sp. Sporidibolus sp. sporobolomyces sp. Sporobolomyces sp.

Rhodotorula spp.

- - Rhodotorula spp.

- - - Rhōdotōōūa sp.

- sporobolomyces sp.

Cryptococcus spp.

- $\bar{R}$ hodosporidium spp.

_ $\ldots \ldots \ldots \ldots 185$

- - - ---.177

- - - - - - 175

- - - - - - 168

- - - - - - 149

- - - - - - 146

$-----\cdots 147$

- Saccharō̄ycōsis spp. Nematospora sp.

- $\ldots$ Nematospora sp. 164

- - - - - - 155

- - - - - - 152

$-----\ldots--153$

- Saccharomycopsis spp.

- Saccharomycopsis sp.

- - - - - - 162

160

- Metschnikowia spp.

159

- Metśs̄nnikowía spp.

- 
Table 4-cont.

Negative

162 Septate hyphae

163 D, L-Lactate growth - -

164 Starch growth

165 Septate hyphae - - -

166 D-Galactose growth - -

167 Raffinose growth

168 myo-Inositol growth $\overline{-}$

169 Erythritol growth _ - -

170 D-Galactose growth - -

171 Sucrose growth _ - -

172 L-Sorbose growth - - -

173 D-Galactose growth - Sucrose growth Cellobiose growth - D-Glucose fermentation D-Mannitol growth Maltose fermentation - Sucrose growth D-Galactose growth - L-Rhamnose growth - Erythritol growth - Raffinose growth - D, L-Lactate growth _ Apical budding Budding cells Erythritol growth Nitrate growth L-Rhamnose growth - Raffinose growth _ - Cellobiose growth - Growth in $60 \%$ D-glucose $\bar{e}$ L-Sorbose growth D-Galactose growth - Citrate growth Ethylamine growth - Growth with $0.01 \%$ cycloheximide

D-Mannitol growth

Glycerol growth

D, L-Lactate growth

D-Glucose fermentation Septate hyphae

D,L-Lactate growth - Ethylamine growth - Growth without niacin Growth with $0.01 \%$ cycloheximide

208 D-Ribose growth

209 Growth without pyridoxine

210 Growth without biotin

211 Glycerol growth

212 D-delta-Gluconoläctoñegrowth

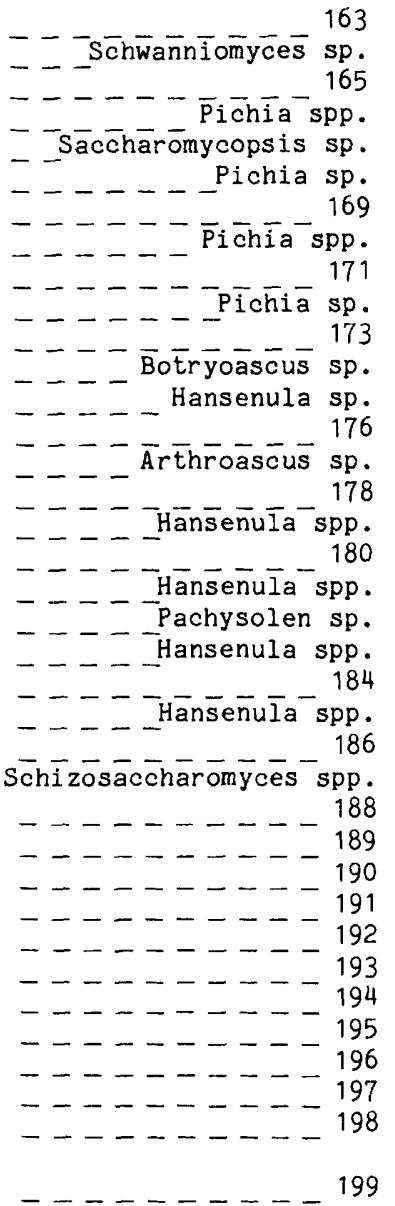

Pichia sp. Sacchäromyces spp. Kluyveromyces $\mathrm{sp}$.

Pichia sp.

- - - - píichia sp.

- - - - - - - - 204

$---\cdots---205$

- - - - - - - - - 206

- - - - - - 207

_. _ Saccharomyces spp.

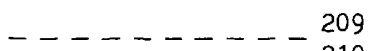

- - - - - - - - 210

Pachytichospora sp. Saccharomyces sp. _. Saccharomyces spp. _ $\ldots$ Saccharomyces sp.
Positive

_ Saccharomycopsis sp.

- $\ldots$ - $\ldots$ Pichia sp. 166

167

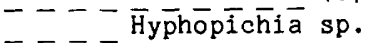

172

- - - - - - - 170

-

Amb $\bar{r}$ osiozyma sp.

_. _ Ambrosiozyma sp.

- - - - Píchia sp.

- - Stēphañoascus sp.

- Hanseniaspora spp.

- - Wickerhamia sp.

- - 179

- - - D Dekkera spp.

- _ - _ - _ _ 182

- - - - - 181

- - - - Hañsenüla sp.

- - - - _ _ _ _ 183

_ _ - Hanseñula spp.

- - - - Hormoascus sp.

- - - - - - - 260

- - - - - - 28187

- - - - - - - 252

- - - - - - - 252

- - - - - - 250

- - - - - - - 224

- - - - - - - 222

- - - - - - 217

- - - - - - - 214

- - - - - - 201

- - - - - $\overline{\text { Pichía }}$ spp.

- - - - Pichia spp.

- - - - - - 200

Pichia sp. Saccharomyces sp. Pichia spp. Saccharomyces sp. Pichia spp.

203

- Guīiliermondē̄la sp.

_ _ _ _ _ _ _ _ 213

- - Saccharomyces sp.

_ _ _ _ _ _ _ _ 208

- Saccharomyces spp.

Kluyveromyces $\mathrm{sp}$. Saccharomyces $\mathrm{sp}$. Kluyveromyces sp. _ $\ldots \ldots \ldots$. $\ldots \ldots \ldots$

Kluyveromyces $\mathrm{sp}$. Saccharomyces $\mathrm{sp}$. 212

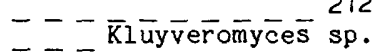


Table 4-cont.

Negative

213 Septate hyphae

214 Septate hyphae

215 Sucrose growth - - - -

216 Starch growth

217 D-Glucose fermentation_

218 Growth without pyridoxine

219 D, L-Lactate growth
Filamentous

Citrate growth

D-Glucose fermentation-

D-Glucose fermentation Starch growth D, L-Lactate growth

Melibiose growth Growth without niacin Ethylamine growth _. L- Sorbose growth__ _L-Sorbose growth__ 2-Ketogluconate $\overline{\text { growth }}$ Growth in $60 \%$ D-glucoseD-Galactose growth Growth with $0.01 \%$ cycloheximide

Glycerol growth D-delta-Gluconolāetonegrowth

\section{Filamentous} Growth without $\overline{\text { niaciñ }} \overline{-}$ Starch growth Cellobiose growth Growth in $60 \% \mathrm{D}-\mathrm{gl}$ ucose Growth with $0.01 \%$ cycloheximide

D-delta-Gluconolactone growth

Growth in 50\% D-glucose Lactose growth Starch growth ${ }^{-}-\ldots$ D-Glucose fermentationStarch formation

Maltose growth Inulin growth Melibiose growth Trehalose fermentationLactose growth_. . . Growth in 50\% D-glucose Raffinose growth
Growth without ${ }^{-} \bar{h}$ amin Cellobiose growt $\bar{h}--$ Sucrose growth L-Rhamnose growth $\overline{\mathrm{n}}$
Saccharomyces spp.

_...... Tichía spp.

_ Lodderomyces sp.

_ _

- - $\overline{\mathrm{K}} \mathrm{l} \overline{\mathrm{u}} \overline{\mathrm{v}}$ eromyces $\mathrm{sp}$. Pichia spp.

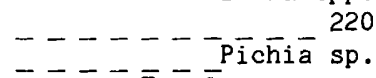

- - Torulaspora sp.

Zygosaccharomyces spp. - - - Torulaspora sp. _ _ _ _ _ _ _ _ 223 _ - L Lipomyces sp. - - Dē̄arȳomyces spp. _ Pichia sp. Lipomyces sp.

_ _ _ _ _ _ - 229

- - - - - - - 230

- - - - - 231

$-\ldots--\ldots-\ldots 232$

- - Saçcharō̄yces sp.

- - - - - - 236

- - - - - 237

_

_ Saccharomyces spp.

_. Saccharomyces sp.

Torulaspora sp.

- KI

- Zy gosaccharomyces sp.

- Z 244

- - - - - - 245

- - - - - - 246

Saccharomyces spp.

_ - Saccharomyces sp.

_ _ _ Saccharomyces sp. 249

- Saccharomyces sp. _ - Wickerhamiella sp.

_ _ - _ _ _ _ _ 253

_ _

- - - - - _ _ 255

_ - Debaryomyces $\overline{s p p}$.

257

_ - Débär yomyces sp.

- - Debaryomyces spp.

Debaryomyces spp. Lipomyces sp. _
_ _ _ _ . _ . 23234

- - - - - - - 235
Positive

Guilliermondella sp. _ $\ldots$ Z $\ldots$ Zndera sp. - - - - - - - - - - Píchia sp. - - - - - P 218

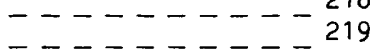
_. _ . _ _ _ _ _ 221

_

Zȳgosaccharomyces spp.

_ $\ldots \ldots$ Pichia spp.

Pichia spp.

- K̄̄uyveromyces spp.

_ _ KIuyveromyces spp.

- - - - - - - - 226

$--------227$

_ - Débaryomyces sp.

- - - - - - - 243

- - - - - - - 233

- K̄̄uyverēomyces spp.

Kluyveromyces sp.

240

- - Törūiaspō̄a spp.

- - Torulaspora sp.

- - - Torulaspora sp.

_

_ _ _ _ _ _ _ 239

_. KI uyveromyces spp.

- Kluyveromyces $\mathrm{sp}$.

- _ - _ _ _ _ 242

- Debaryomyces sp.

_. - - _ _ _ 248

- - To $\overrightarrow{r u l a s p o ̄ r a ~ s p . ~}$

-zȳgosaccharomyces spp.

- $\ldots \ldots \ldots 247$

Zygosaccharomyces $\mathrm{sp}$. - Debaryomyces spp.

- Debaryomyces sp.

_ - Debaryomyces spp.

- - Citeromyces sp.

- - Lipomyces spp.

- 254

- - - - - - 259

- - - - - - - 256

- - - - - - 258

- - - - Wingea sp.

- - Debaryomyces sp.

Pichia sp. Debaryomyces spp.

261

- Saccharō̄ō̄odes sp. 
greater detail than that required for the key in Table 4, needing more expert knowledge and experience. The physiological tests which constitute the greatest part of our key are also less time-consuming than microscopical examinations and can be carried out by a less experienced operator. Moreover, the key in Table 4 does not require examination of the sexual cycle of the basidiomycetous species.

However, in the same way that a key for identifying species cannot identify new species not in the key, the key in Table 4 cannot be applied to species other than those listed by Barnett $e t$ al. (1979). Any new species will be assigned to the genus of a species whose test results in the key are identical to those of the new species, but this may not give the correct genus as the genera are not classified in terms of the characteristics used in the key. However, the key is still only marginally less useful than that of Lodder (1970), in view of the many recent changes in generic characteristics and the newly invented genera, to which Lodder's key also cannot apply.

\section{A P PENDIX 1}

\section{Irredundant test sets to identify groups of taxa}

Barnett et al. (1979) used a method (reviewed by Payne \& Preece, 1980) that determined all irredundant test sets available to identify the yeast species in a particular key. This method can be adapted to form sets to identify groups instead of individual taxa. In the first stage a triangular array is formed and in the $(i, j)$ th position $(i<j)$ are listed the tests that can distinguish the pair of taxa, $i$ and $j$. To identify groups instead of individual taxa, array entries are omitted where each member of the pair of taxa is from the same group. Table 5 contains the appropriate array for the groups in Table 1; taxa C and D have different results with tests 1 and 5, thus the $(3,4)$ th entry is ' 1,5 '. Any entries that contain other entries are deleted. For example, as ' 4,5 ' is an entry in Table $5,{ }^{\prime} 1,3,4,5$ ' $, 3,4,5$ ' etc. are deleted. [Such entries can be omitted since, in order to distinguish between the pair of taxa A and D, corresponding to entry ' 4,5 ' in Table 5 , either test 4 or test 5 must be in the irredundant set. These tests ( 4 and 5 ) will also distinguish between the pairs of taxa corresponding to entries ' $1,3,4,5$ ' etc.] The deleted entries in Table 5 are enclosed in brackets.

The surviving entries are then expressed as a sum and are multiplied together according to the Boolean rules $i i=i$ and, for example, $i j+i j k=i j$.

For example, from Table 5,

$$
\begin{aligned}
& (4+5)(2+4)(1+4)(1+6)(1+5)(3+5)(1+2)(2+5)(2+6)(3+6)(5+6) \\
= & (24+44+25+45)(1+4)(1+6) \ldots \\
= & (4+25)(1+4)(1+6) \ldots
\end{aligned}
$$

Each term in the first bracket is a set of tests that can distinguish between taxa $A$ and $D$ and taxa $A$ and $G$. (This indicates the rationale of the rules. ' 44 ' becomes ' 4 ' because there is no need to record a test more than once in a set. The fact that ' 4 ' is a set in its own right implies that ' 24 ' and ' 45 ' contain redundant tests 2 and 5 , respectively; if these are deleted two more instances of set ' 4 ' would be obtained; there is no need to record a set more than once so ' 24 ' and ' 45 ' can be deleted.) Once all the brackets have been multiplied together the sets each contain tests to distinguish all the pairs of taxa belonging to different groups, and if any test in one of the sets is deleted there will be some pair of taxa in different groups that can no longer be distinguished.

Continuing the multiplication from Table 5 ,

$$
\begin{aligned}
& (4+25)(1+4)(1+6)(1+5)(3+5)(1+2)(2+5)(2+6)(3+6)(5+6) \\
= & (4+125)(1+6) \ldots \\
= & (14+46+125)(1+5) \ldots \\
= & (14+456+125)(3+5) \ldots \\
= & (134+145+456+125)(1+2) \ldots \\
= & (134+145+2456+125)(2+5) \ldots \\
= & (1234+145+2456+125)(2+6) \ldots \\
= & (1234+1456+2456+125)(3+6) \ldots \\
= & (1234+1456+2456+1235+1256)(5+6) \\
= & (12346+1456+2456+1235+1256)
\end{aligned}
$$

There are five possible irredundant sets of tests, namely, 12346, 1456, 2456, 1235 and 1256 . These sets cannot be obtained from the group data in Table 1 as there is no single test that can distinguish any of the pairs of groups. 
Table 5. Lists of tests that can distinguish the taxa of Table 1 that are in different groups

$\begin{array}{lccccccccccc}\text { Taxon } & \text { Group } & \text { A } & \text { B } & \text { C } & \text { D } & \text { E } & \text { F } & \text { G } & \text { H } & \text { I } \\ \text { A } & \text { I } & - & - & - & 4,5 & (1,3,4,5) & (3,4,5) & 2,4 & (2,5,6) & (2,3,4,6) \\ \text { B } & \text { I } & & - & - & (1,2,4) & (2,3,4) & (1,3,4) & 1,4 & 1,6 & (1,3,4,6) \\ \text { C } & \text { I } & & & - & 1,5 & 3,5 & (1,3,5) & 1,2 & (1,2,5,6) & (1,2,3,6) \\ \text { D } & \text { II } & & & & - & - & - & 2,5 & 2,6 & (2,3,5,6) \\ \text { E } & \text { II } & & & & & - & - & (1,2,3,5) & (1,2,3) & (1,2,5) \\ \text { F } & \text { II } & & & & & & - & (3,5) & 3,6 & 5,6 \\ \text { G } & \text { III } & & & & & & & - & - & - \\ \text { H } & \text { III } & & & & & & & & - & - \\ \text { I } & \text { III } & & & & & & & & & -\end{array}$

See Appendix 1 for meaning of entries in parentheses.

When only a single irredundant set is required, it may be formed sequentially by choosing tests one at a time. Payne \& Preece (1980) reviewed criteria for deciding which test to include at each stage. Many of these criteria involve the separation coefficient of Gyllenberg (1964). This is the number of pairs of taxa $(i, j)$ that are distinguished either by the current test, or by some test already in the set. To select tests to distinguish groups instead of individual taxa, only pairs of taxa belonging to different groups need to be considered.

\section{AP P ENDIX 2}

\section{Criterion functions for selecting tests to identify groups of taxa}

Selection criterion functions, designed to select tests to identify individual taxa, require modification to select tests to identify groups of taxa. An exception is the function of Dallwitz (1974), whose program allows intra-taxon variability to be expressed by specifying more than one 'item' for a taxon. Thus, each taxon may itself be a group of several 'items'. Most taxa will, however, consist of a single 'item' so, at any point in the key, there will be few (if any) tests that are variable for all the groups of 'items' which occur there; Dallwitz's function does not satisfactorily distinguish between tests with this much variability. For yeast genera, however, so many results are variable that such tests can be expected to occur at many points of the key. The two functions derived below can cope with such tests.

The first function is obtained by modifying the function $M_{\mathrm{v}}$ of Payne (1981):

$$
\left(M_{\mathrm{v}}\right)_{i}=-\sum_{k=1}^{m_{i}}\left\{\left(p_{i k}+r_{i} / m_{i}\right)\left(1-r_{i}-p_{i k}\right)\right\}
$$

where $m_{i}$ is the number of possible results to test $i, p_{i k}$ is the proportion of taxa in the current subset that always give result $k$ to test $i$, and $r_{i}$ is the proportion of taxa in the current subset with variable results to test $i$. This is an extension, to tests with more than two possible results, of the function $D V$ derived by Morse (1971) from Gyllenberg's Separation Coefficient. The term in the first bracket is the proportion of specimens that give result $k$ to test $i$ assuming that, for each variable taxon, there is an equal probability $\left(1 / m_{i}\right)$ of obtaining results, $1,2, \ldots, m_{i}$. The second term is the proportion of specimens belonging to taxa that cannot give result $k$. Thus $\left(M_{v}\right)_{i}$ is minus the proportion of pairs of taxa separated (either wholly or partially) by test $i$. For identifying groups, this function should become minus the proportion of pairs of taxa in different groups separated by the test; that is

$$
\begin{aligned}
\left(G_{v}\right)_{i}=- & \sum_{k=1}^{m_{i}} \sum_{j=1}^{n}\left[\left(q_{i j k}+s_{i j} / m_{i}\right) \times \sum_{i \neq j}\left\{\left(\sum_{k=1}^{m_{i}} q_{i l k}\right)-q_{i l k}\right\}\right] \\
=- & \sum_{k=1}^{m_{i}} \sum_{j=1}^{n} q_{i j k}^{2}+\sum_{k=1}^{m_{i}}\left(\sum_{j=1}^{n} q_{i j k}\right)^{2}+\sum_{j=1}^{n}\left(\sum_{k=1}^{m_{i}} q_{i j k}\right)^{2}-\left(\sum_{k=1}^{m_{i}} \sum_{j=1}^{n} q_{i j k}\right)^{2} \\
& +\left(1-1 / m_{i}\right)\left\{\sum_{j=1}^{n}\left(s_{i j} \times \sum_{k=1}^{m_{i}} q_{i j k}\right)-\left(\sum_{j=1}^{n} s_{i j}\right) \times\left(\sum_{k=1}^{m_{i}} q_{i j k}\right)\right\}
\end{aligned}
$$


where $n$ is the number of groups, $q_{j k}$ the proportion of taxa in the current subset that are from group $j$ and that always give result $k$ to test $i$, and $s_{i}$ is the proportion of taxa from group $j$ that have variable results.

The second function, which was used to construct the key to yeast genera in Table 4, is derived from the function $M_{\mathrm{e}}$ of Brown (1977). This selects the test for which the expected entropy of the posterior probabilities of the taxa, given the result of the test, is minimum. Thus, the aim when selecting each test is to make the probabilities that the specimen belongs to each taxon as different as possible. This will be achieved when, for each result, all the probabilities except one are zero; that is, when the subsets formed by the test all contain only one taxon. The derivation, like that of $M_{v}$, assumes that equal proportions of variable taxa give each result.

$$
\left(M_{\mathrm{e}}\right)_{i}=\sum_{k=1}^{m_{i}}\left\{\left(p_{i k}+r_{i} / m_{i}\right) \log \left(p_{i k}+r_{i} / m_{i}\right)\right\}+r_{i} \log m_{i}
$$

[This is the negative of the function of Brown (1977), who defined the best test to be that with maximum function value.] Under the same assumptions, the expected entropy of the posterior probabilities of the groups is given by

$$
\left(G_{\mathrm{e}}\right)_{i}=-\sum_{k=1}^{m_{i}}\left[t_{i k} \times \sum_{j=1}^{n}\left[\left\{\left(q_{i j k}+s_{i j} / m_{i}\right) / t_{i k}\right\} \log \left\{\left(q_{i j k}+s_{i j} / m_{i}\right) / t_{i k}\right\}\right]\right]
$$

where $t_{i k}=\sum_{j=1}^{n}\left(q_{i j k}+s_{i j} / m_{i}\right)$. Thus,

$$
\left(G_{\mathrm{e}}\right)_{i}=\sum_{k=1}^{m_{i}}\left\{\sum_{j=1}^{n}\left(q_{i j k}+s_{i j} / m_{i}\right)\right\} \log \left\{\sum_{j=1}^{n}\left(q_{i j k}+s_{i j} / m_{i}\right)\right\}-\sum_{k=1}^{m_{i}} \sum_{j=1}^{n}\left\{\left(q_{i j k}+s_{i j} / m_{i}\right) \log \left(q_{i j k}+s_{i j} / m_{i}\right)\right\}
$$

An alternative justification for $M_{\mathrm{e}}$ (Payne \& Preece, 1980; Payne, 1981) uses the noiseless coding theorem of Shannon (1948) to relate $\left(M_{\mathrm{e}}\right)_{i}$ to the expected number of tests required to complete the key after test $i$, assuming that this is done optimally. This enables $M_{\mathrm{e}}$ to be extended to tests with different costs. However, this would be less convincing for $G_{\mathrm{e}}$ because to complete a key to the groups in an optimal way requires tests to be available that have constant results within each group.

The assumption that equal proportions of variable taxa give each result, which greatly simplifies the algebraic form of $M_{\mathrm{v}}, G_{\mathrm{v}}, M_{\mathrm{e}}$ and $G_{\mathrm{e}}$, is not crucial. If it were badly wrong, the test selected might not be the best available and the resulting key might be less efficient; however, the identifications obtained would still be correct. In most situations the assumption will be reasonable - either because the probabilities are known to be nearly equal, or because (as with the yeasts) there is not sufficient information to contradict it. However, if estimates of the probabilities are available, the functions can easily be modified. For example, the full form of $M_{\mathrm{e}}$ is given in equations (4) and (5) of Payne (1981).

Use of the expected entropy of the posterior probabilities of the taxa to select test for probabilistic identification, has been discussed by, for example, Good (1970), Moiseeva \& Usov (1969), Taylor (1970), Knill-Jones et al. (1973) and Payne (1975). Other functions used for this purpose can be adapted similarly.

\section{REFERENCES}

Barnett, J. A. \& Pankhurst, R. J. (1974). A New Key to the Yeasts. Amsterdam: North-Holland Publishing Co.

BARNeTT, J. A., BASCOMB, S. \& Gower, J. C. (1975). A maximal predictive classification of Klebsielleae and of the yeasts. Journal of General Microbiology 86, 93-102.

Barnett, J. A., Payne, R. W. \& Yarrow, D. (1979). $A$ Guide to Identifying and Classifying Yeasts. Cambridge: Cambridge University Press.

Brown, P. J. (1977). Functions for selecting tests in diagnostic key construction. Biometrika 64, 589596.
Dallwitz, M. J. (1974). A flexible computer program for generating identification keys. Systematic Zoology 27, 50-57.

Good, I. J. (1970). Some statistical methods in machine-intelligence research. Mathematical Biosciences 6, 185-208.

Gower, J. C. (1973). Classification problems. Bulletin of the International Statistical Institute 44, 296301.

Gower, J. C. (1974). Maximal predictive classification. Biometrics 30, 643-654.

GyllenBerg, H. A. (1964). An approach to numerical description of biological populations. Annales 
Academiae scientiarum fennicae, Series A IV, Biological Part 81, 1-23.

Knill-Jones, R. P., Stern, R. B., Girmes, D. H., Maxwell, J. D., Thompson, R. P. H. \& Williams, R. (1973). Use of sequential Bayesian model in diagnosis of jaundice by computer. British Medical Journal 1, 530-534.

Kreger-vaN Ru, N. J. W. (1970a). Debaryomyces Lodder et Kreger-van $\mathrm{Rij}$ nom. conserv. In The Yeasts. A Taxonomic Study, pp. 129-156. Edited by J. Lodder. Amsterdam: North-Holland Publishing Co.

Kreger-van RiJ, N. J. W. (1970 b). Pichia Hansen. In The Yeasts. A Taxonomic Study, pp. 455-554. Edited by J. Lodder. Amsterdam: North-Holland Publishing Co.

LODDER, J. (1970). Introduction to the chapters IV, V, VI and VII, and key to the genera. In The Yeasts. $A$ Taxonomic Study, pp. 114-120. Edited by J. Lodder. Amsterdam: North-Holland Publishing Co.

Morseeva, N. I. \& Usov, V. V. (1969). Some medical and mathematical aspects of computer diagnosis. Proceedings of the Institution of Electrical and Electronics Engineers 57, 1919-1925.

MORSE, L. E. (1971). Specimen identification and key construction with time-sharing computers. Taxon 20, 269-282.
PANkhuRst, R. J. (1970). A computer program for generating diagnostic keys. Computer Journal 13, 145-151.

PAYNE, R. W. (1975). Genkey: a program for constructing diagnostic keys. In Biological Identification with Computers, pp. 65-72. Edited by R. J. Pankhurst. London: Academic Press.

PAYNe, R. W. (1978). Genkey: a Program for Constructing and Printing Identification Keys and Diagnostic Tables. Harpenden: Rothamsted Experimental Station.

PAYNe, R. W. (1981). Selection criteria for the construction of efficient diagnostic keys. Journal of Statistical Planning and Inference 5, 27-36.

PAYNe, R. W. \& PREece, D. A. (1980). Identification keys and diagnostic tables: a review (with discussion). Journal of the Royal Statistical Society, Series A 143, 253-292.

Payne, R. W., Walton, E. \& Barnett, J. A. (1974). A new way of representing diagnostic keys. Journal of General Microbiology 83, 413-414.

ShanNon, C. E. (1948). A mathematical theory of communication. Bell System Technical Journal 27, 379-423, 623-656.

TAYLOR, T. R. (1970). Computer-guided diagnosis. Journal of the Royal College of Physicians of London 4, 188-194. 